\title{
Sentido y alcances prácticos de la Educación Física Escolar como un Derecho Humano. Análisis en las Bases Curriculares chilenas de Educación Básica
}

\author{
María Angélica Oliva ${ }^{\mathrm{a}, \mathrm{b}, \mathrm{c} *} \mathbb{C}_{\mathbb{C}}$, Hernaldo Carrasco Beltrán $\mathrm{n}^{\mathrm{b}, \mathrm{c}(\mathbb{C})}$
}

\section{Palavras Chave:}

Derechos Humanos; Educación Física Escolar; Bases Curriculares de Chile; Educación Básica.

\section{RESUMEN}

Resumen Se analiza la Educación Física Escolar como un Derecho Humano en las Bases Curriculares de Educación Básica en Chile. Se utiliza una metodología hermenéutica/dialéctica en 2 corpus -Bases Curriculares Nacionales de Educación Física y Salud y marco jurídico de Derechos Humanosmediante una estrategia documental y un análisis de contenido inductivo. Se concluye que la Educación Física Escolar podría constituir un Derecho Humano, por su defensa de la dignidad, no discriminación, respeto a la diversidad, entre otros. Sin embargo, en el proceso de diseño curricular los Derechos Humanos pierden la presencia que poseen en las Bases Curriculares Nacionales, debido a la circunstancia política de su enunciación y a la carencia de un Enfoque Basado en Derechos Humanos (EBDH).

\section{RESUMO}

A Educação Física Escolar é analisada como um Direito Humano nas Bases Curriculares da Educação Básica no Chile. A metodología escolhida é a hermenéutica/dialética aplicado a 2 corpus: Bases Curriculares Nacionais para a Educação Física e Saúde e o marco jurídico dos Direitos Humanos, através de: análise documental, análise indutiva de conteúdo. Conclui-se que a educação física escolar pode constituir um direito humano, devido à sua defesa da dignidade, da não discriminação, do respeito pela diversidade, entre outros. No entanto, no processo de desenho do currículo, os Direitos Humanos perdem sua presença nas Bases Curriculares Nacionais, devido à circunstância política de seu enunciado e à falta de uma Abordagem Baseada nos Direitos Humanos (ABDH).

\section{Keywords:} Human Rights; School Physical Education Chilean Curriculum Foundations; Primary Education.

\begin{abstract}
School Physical Education is analyzed as a Human Right in the Curriculum Foundations for Primary Education in Chile. A hermeneutical/dialectical methodology is applied in 2 text corpus - National Curriculum Foundations for both Physical Education and Health and the legal framework of Human Rights - to which they are applied: document-like strategy, inductive content analysis. It is concluded that School Physical Education could constitute a human right, for his defense of the dignity, nondiscrimination, and respect to diversity, among other categories. However, in the curriculum design process, Human Rights lose their presence in the National Curricular Bases, due to the political circumstance of their enunciation and the lack of a Human Rights-Based Approach (HRBA).
\end{abstract}

\footnotetext{
a Instituto Interuniversitario de Investigación Educativa (IESED-Chile), Santiago, Chile.

${ }^{b}$ Centro de Estudios Avanzados (CEA), Santiago, Chile.

' Universidad de Playa Ancha (UPLA), Valparaíso, Chile.
}

* Autor correspondente: María Angélica Oliva

E-mail: angelica.oliva@upla.cl 


\section{INTRODUCCIÓN}

Este artículo sitúa la díada Educación Física Escolar y Derechos Humanos en el lugar de la interrogación para analizar su sentido y alcances prácticos en las Bases Curriculares de Chile. El presupuesto de la interrogación está en el significado de la díada como unión o intersección de dos principios o conjuntos que se complementan recíprocamente.

En el centro de la intersección, es posible emplazar la idea de dignidad fuente de todos los Derechos Humanos y categoría que fundamenta su invisibilidad (ONU, 2015; Habermas, 2010). Por su parte, la práctica de la Educación Física, la Actividad Física y el Deporte constituye, para la UNESCO (2015), un Derecho Fundamental.

El artículo se sustenta en una investigación que utiliza el Enfoque Basado en los Derechos Humanos [EBDH], para indagar en el sentido y alcances del Derecho a la Educación para examinar el discurso de la calidad de la educación en la política educativa chilena reciente.

Se considera el Derecho Internacional de los Derechos Humanos como un marco normativo para orientar el diseño, implementación, monitoreo y evaluación de políticas públicas, analizar los problemas sociales y las condiciones de desigualdad e identificar las distribuciones inequitativas de poder que dificultan el desarrollo. Este marco, posee una concreción en las obligaciones del Estado en Derechos Humanos de: respetar, proteger, garantizar, y de satisfacer o tomar medidas (OACNUDH, 2006).

El artículo dialoga con las perspectivas práctica, crítica y postcrítica y se orienta hacia las funciones sociales y educativas de la escuela y sus disciplinas escolares (Fernández Enguita, 1997). Dentro de ellas, la Educación Física, cuya historia ha estado ligada a un modelo curricular tecnocrático y biomédico (Moreno, 2018).

Se profundiza en el nexo entre dignidad y Educación Física orientada hacia la promoción de valores y actitudes, tales como, el respeto a las personas y la igualdad "transformar la Educación Física en una asignatura capaz de favorecer prácticas positivas y beneficiosas de actividad física para los más necesitados de los beneficios de éstas" (Moreno, Campos, Almonacid, 2012, p.24).

Los Derechos Humanos corresponden al conjunto de los derechos individuales y colectivos inherentes a todas las personas sin distinción; son universales, inalienables, indivisibles e interdependientes (OACNUDH, 2010); regulan la obligación del Estado en materia de: cobertura, accesibilidad, aceptabilidad y calidad (DESC, 1999; Tomasevski, 2004).
Para la comprensión del sentido y alcances prácticos de la Educación Física Escolar como Derecho Humano, resulta óptimo atender a la estructura tríadica del derecho: hecho histórico-cultural, norma y valor, que constituye la experiencia jurídica (Reale, 1997). Las principales declaraciones en favor de los Derechos Humanos, la Actividad Física y el Deporte, emanan del marco jurídico de los Derechos Humanos; se concretan en cartas, actas y convenciones generales que reafirman el estatuto de la Educación Física y el Deporte. El hecho histórico-social, refiere al ser humano y su dignidad, sujeto de una educación escolarizada. La Educación Física, la Actividad Física y el Deporte, constituye un ámbito de esa dignidad, que concreta "la moral del respeto igualitario de toda persona con el derecho positivo y el proceso de legislación democrático, de tal forma que su interacción puede dar origen a un orden político fundado en los derechos humanos" (Habermas,2010, p.10). Manifestaciones del marco normativo que regula la Educación Física Escolar como Derecho Humano son, por vía ejemplar: la Carta Europea del Deporte (1992), resalta que el deporte promueve el desarrollo personal y social, y el ejercicio físico contribuye al equilibrio fisiológico y psicológico del ser humano; el Consejo Europeo de Niza (2000), reconoce al deporte como una actividad humana basada en unos valores sociales educativos y culturales esenciales; la Carta de Toronto para la Promoción de la Educación Física (2010), destaca su valor en la promoción del bienestar, la salud física, mental, previene enfermedades y mejora la calidad de vida, en fin, la Declaración de Berlín (2013), determina que la Educación Física Escolar es el medio más efectivo para proporcionar a todos los niños y jóvenes competencias, aptitudes, actitudes, valores, conocimientos y comprensión para su participación en la sociedad a lo largo de la vida. Finalmente, la dimensión axiológica es transversal a la experiencia jurídica, luego, a los Derechos Humanos, todo lo cual establece un poderoso vínculo con la educación escolarizada. Porque la educación escolarizada, es el ámbito de la Educación Física que, en su condición de disciplina escolar, sujeta saberes y sujetos a un conjunto de normas explícitas e implícitas (simbólicas) que traducen, reproducen y producen orden social dominante (Varela, 1995; Oliva, 2019). La configuración de la Educación Física como disciplina escolar "ha transitado por diversas orientaciones, desde propósitos de desarrollo de la población en cuanto salubridad y productividad, militarización, higiene y seguridad, deportivización, hasta ser considerado un derecho para todas y todos" (Moreno, Gamboa, Poblete, 2014, p.423). 
¿Qué justifica este estudio? La tridimensionalidad del derecho que circunscribe la díada Derechos Humanos y Educación Física Escolar proporciona una respuesta. Repárese en la vulneración de los Derechos Fundamentales de niños y niñas y jóvenes que muestran los resultados de la última Encuesta Nacional de Salud (2016-2017), que arrojan alarmantes cifras con respecto a la actividad física practicada con cierta regularidad, estableciendo parámetros de sedentarismo que ascienden a un $86,7 \%$ del universo consultado asimilable al total país (MINSAL, 20162017). Los resultados de la franja etaria consultada más cercana a la edad escolar (15-19 años) indican que el sedentarismo es de un $73,5 \%$, cifra que debiese ser motivo de preocupación, pues la falta de acceso al derecho de realizar actividad física implica directamente en la promoción de enfermedades no transmisibles, así como de obesidad y otros males degenerativos, repercutiendo en el desarrollo integral de niños y niñas, lo que expresa el vínculo entre Derechos Humanos y Educación Física Escolar.

\section{MATERIAL Y MÉTODOS}

La investigación considera presupuestos ontológicos, epistemológicos y metodológicos, que alcanzan a cualquier investigación científica. Se desarrolla una investigación cualitativa inscrita en el construccionismo y su metodología hermenéutica/ dialéctica (Guba y Lincoln, 1994). Este compromiso investigativo se funda en la distinción diltheyana entre las ciencias de la naturaleza y las ciencias del espíritu y se inscribe en estas últimas - su radical interés por la comprensión, antes que por su explicación- permite explorar, describir y, ciertamente, valorar, tanto la dimensión explícita, manifiesta, 'visible', como inferir la dimensión implícita, subyacente o latente (Dilthey, 2007). Ello se expresa en la opción por la técnica de análisis de contenido cualitativo, cuya fase hermenéutica descubre la tensión entre las dimensiones explícitas e implícitas de un problema. Además, del emplazamiento del problema en el currículum, que refiere al conocimiento que circula, se distribuye y se evalúa en la institución escolar y a la existencia de un código curricular donde es posible inferir un ámbito subyacente (Lundgren,1997). Relacionado, también, con la existencia de un currículum oculto, o significado latente en los diversos elementos de la vida escolar (Jackson, 1998).

La estrategia basada en la documentación (Valles, 1999), es el utillaje para la revisión del corpus normativo y de la literatura. Por su parte, el trabajo empírico se aborda mediante el análisis de contenido de carácter inductivo, que comprende y desarrolla conceptos partiendo de pautas de datos, antes que recogiendo datos para evaluar hipótesis o teorías preconcebidas (Taylor y Bogdan, 1986). En concordancia con la dimensión ontológica y epistemológica que sustenta una investigación, donde la experiencia -el mundo empírico- es el único punto de partida válido a las ciencias del espíritu y a su concepto de sentido (Dilthey, 2007).

La pregunta ¿Qué elementos del mundo empírico constituyen las fuentes de datos en esta investigación?, permite presentar a 2 corpus: Bases Curriculares Nacionales 1ㅇ a 60 básico y el marco jurídico de los Derechos Humanos (Figure 1). Las Bases Curriculares Nacionales promulgadas mediante la Ley General de Educación[LGE] (MINEDUC, 2009), que deroga la Ley Orgánica Constitucional de Enseñanza [LOCE] (MINEDUC, 1990).

Las Bases Curriculares Nacionales corresponden al currículum oficial que prescribe los mínimos y directrices para un sistema educativo o para un nivel del mismo, regulado por instancias políticas y administrativas que enmarcan las prácticas escolares (Gimeno Sacristán, 1995).

La Ley General de Educación, reafirma la naturaleza mixta del sistema escolar chileno instituida en la dictadura cívica militar mediante la Constitución de 1980, que consagra el rol subsidiario el Estado. Su corolario el traspaso de la gestión de los establecimientos escolares, desde la dependencia centralizada a una descentralizada, y la creación de establecimientos de distinto nivel de dependencia: municipal subvencionado, particular subvencionado y particular pagado. Esta estructura se concreta por la transformación en el sistema de financiamiento, desde un subsidio a la oferta a un subsidio de la demanda, que estimula la incorporación masiva de agentes privados y la creación de un mercado de la educación (Oliva, 2008).

En este contexto, se regula una política curricular que posee una parte obligatoria y común, que dispone para todos los establecimientos el cumplimiento de objetivos de aprendizaje establecidos en las Bases Curriculares Nacionales; y una parte diferenciada, que se desmarca de ese currículum común, mediante 3 elementos imbricados: programas de estudio; tiempo de libre disposición; $y$, planes de estudio. Los programas de estudio se construyen por el MINEDUC, desde las Bases Curriculares Nacionales, sin embargo, cada establecimiento decide si aplica estos programas elaborados por MINEDUC o elabora programas propios. El tiempo de libre disposición, corresponde al $30 \%$ del tiempo de la jornada escolar completa que los establecimientos pueden disponer 
Convención Internacional sobre la Eliminación de Todas las Formas de Discriminación Racial [CERDN]

Declaración sobre la Protección de Todas las Personas contra la Tortura y Otros Tratos o Penas Crueles, Inhumanos o Degradantes

Figure 1 . Corpus de Derechos Humanos. Fuente: Producido por los autores

para profundizar o completar los objetivos de las Bases Curriculares Nacionales. Si los establecimientos optan por utilizar los programas de estudio que proporciona el Ministerio de Educación, igualmente pueden contar con un $15 \%$ del tiempo de libre disposición para los mismos efectos. Dentro de otras opciones, el tiempo de libre disposición puede ser destinado a actividades deportivas (MINEDUC, 2018). Los planes de estudio, refieren al tiempo mínimo expresado en horas pedagógicas anuales, que se estima necesario asignar a cada asignatura para cumplir satisfactoriamente con los Programas de estudio propuestos por el MINEDUC (2018).

El corpus de Derechos Humanos, comprende un conjunto de instrumentos protectores de los Derechos Humanos, seleccionados según criterios de exhaustividad y amplitud, incluyendo aquellos que consideran aspectos generacionales, interculturales, de género, tanto de orden internacional, como regional. De ellos, se selecciona los artículos atingentes a las obligaciones del Estado y países firmantes en materia educativa.
Se trabaja con 48 nodos para el corpus de Derechos Humanos y 31 nodos para el corpus de las Bases Curriculares. A continuación, se consulta en el programa Nvivo el porcentaje de cobertura de los nodos, atendiendo a los conceptos destacados de cada corpus. En cuanto al corpus de Derechos Humanos, de un total de 48 nodos, se rastrea su porcentaje de cobertura, cuyo límite superior es de $4,99 \%$ y el límite inferior es $0,06 \%$. Se realiza una selección para graficar las variables observadas que evidencian los primeros 10 nodos, correspondiente a: igualdad de acceso a la educación; fortalecimiento del respeto de los Derechos Humanos; amistad entre naciones; fortalecimiento del respeto de las libertades; gratuidad; libertad de padres y tutores legales para elegir el tipo de educación que recibirán niños y niñas y adolescentes; dignidad humana; convivencia armónica en una sociedad libre; comprensión y tolerancia.

Para delimitar el corpus de las Bases Curriculares Nacionales se selecciona el primer apartado de Antecedentes y la sección completa de las Bases Curriculares de Educación Física y Salud. En este corpus, 
se trabaja con 31 nodos; los primeros 10, a pesar de su bajo porcentaje de cobertura son: calidad de la enseñanza; capacidad de comunicación; capacidad de reflexión; formación integral; igualdad de acceso a la educación; desarrollo de autonomía; pensamiento crítico; respeto a la diversidad; desarrollo pleno de la personalidad; y dignidad humana (Figure 2). Dentro de las categorías rastreadas, destaca el principio de igualdad en más de un nodo, relacionado con los principios de: acceso a la educación (18 referencias, cobertura de 4,99\%); igualdad de oportunidades (6 referencias, cobertura de 1,56\%); e igualdad de trato ( 2 referencias, cobertura de $0,50 \%$ ). En suma, el principio de igualdad alcanza un $7,05 \%$ de cobertura a nivel global del corpus de Derechos Humanos.

Posteriormente, se utiliza una de las estrategias básicas del software NVivo: la creación de un diagrama de intersección con los nodos presentes en ambos corpus. El programa arroja una matriz de intersección que permite observar las categorías vinculadas, tanto a nivel global como particular, en cada fuente de datos utilizada. Para efectos de este estudio, se focaliza el análisis en los nodos comunes en ambos corpus).

\section{RESULTADOS Y DISCUSIÓN}

La díada Educación Física Escolar-Derechos Humanos, inscrita en la tridimensionalidad de los Derechos Humanos, permite organizar los siguientes resultados de la investigación (Figure 3 ).

1.La existencia de una díada o intersección entre Derechos Humanos y Educación Física Escolar. Sus elemenos comunes corresponden a 7 categorías, dentro de éstas: las más relevantes son: principio de igualdad, defensa de la dignidad humana, desarrollo pleno de la personalidad, calidad de la enseñanza y respeto por la diversidad. Se reafirma la dignidad humana como piedra angular de los Derechos Humanos, en consonancia con una educación que tiene por objeto contribuir al desarrollo integral de todas las personas en sus dimensiones espiritual, ética, moral intelectual, artística y física (MINEDUC, 2018).

No obstante esta evidencia, en su conjunto las Bases Curriculares para la Educación Física Escolar no se fundamentan en un Enfoque Basado en Derecho Humanos [EBDH], a contrario sensu, de la Política Nacional de Actividad Física y Deporte diseñada en un Enfoque de EBDH.

Un elemento para discutir este resultado, está en la dimensión contextual de la díada, indispensable en un estudio cualitativo. Dentro de un conjunto de variables, puede destacarse la racionalidad técnicoinstrumental, enfoque predominante de las Bases Curriculares Nacionales, funcional a la reproducción y desigualdad social (Oliva, 2017).

2. Díada Educación Física Escolar-Derechos Humanos: en el trasfondo de lo explícito, lo subyacente (implícito). Nótese cómo el eje habilidades motrices: locomotrices, manipulativas, de estabilidad, plantea su relevancia para el desarrollo de procesos cognitivos $y$, a su paso, las actitudes integradas a conocimientos y habilidades de la asignatura. El eje vida activa y saludable, considera de máxima importancia que toda la población pueda acceder a una práctica física deportiva; el juego es un vehículo de socialización, construcción de lazos, respeto a las reglas, cooperación y autonomía, aspectos relacionados con los Derechos Humanos. Finalmente, el eje de seguridad, juego limpio y liderazgo, destaca las normas de seguridad fundamentales para la prevención de accidentes en la práctica de actividades físicas y/o deportivas. Se considera necesario la atención transversal a la diversidad y empatía, que reconoce el carácter único de cada ser humano (MINEDUC, 2018).

Por su parte, las Bases Curriculares Nacionales en su eje actitudes, plantean 8 actitudes a desarrollar mediante la Educación Física: valorar los efectos

\begin{tabular}{|c|c|c|c|}
\hline Nodo/ Categoría presente & \multicolumn{2}{|c|}{$\begin{array}{l}\text { Porcentaje de cobertura } \\
\text { Corpus Bases curriculares }\end{array}$} & \multirow{2}{*}{$\begin{array}{l}\text { Porcentaje de cobertura } \\
\text { Corpus Derechos Humanos } \\
0,75 \%\end{array}$} \\
\hline Respeto a la diversidad & - & $0,3 \%$ & \\
\hline Igualdad de acceso a la educación & - & $0,33 \%$ & $4,99 \%$ \\
\hline Fortalecimiento respeto de las libertades & $\bullet$ & $0,22 \%$ & $2,41 \%$ \\
\hline Desarrollo pleno de la personalidad & - & $0,22 \%$ & $1,79 \%$ \\
\hline Calidad de la enseñanza & - & $0,45 \%$ & $0,36 \%$ \\
\hline Dignidad humana & - & $0,22 \%$ & $1,96 \%$ \\
\hline Tolerancia & - & $0,09 \%$ & $1,9 \%$ \\
\hline
\end{tabular}

Figure 2. Intersección de categorías DDHH y Bases Curriculares Nacionales EFS. 1ำ a 6을ásico. Fuente: Producido por los autores 


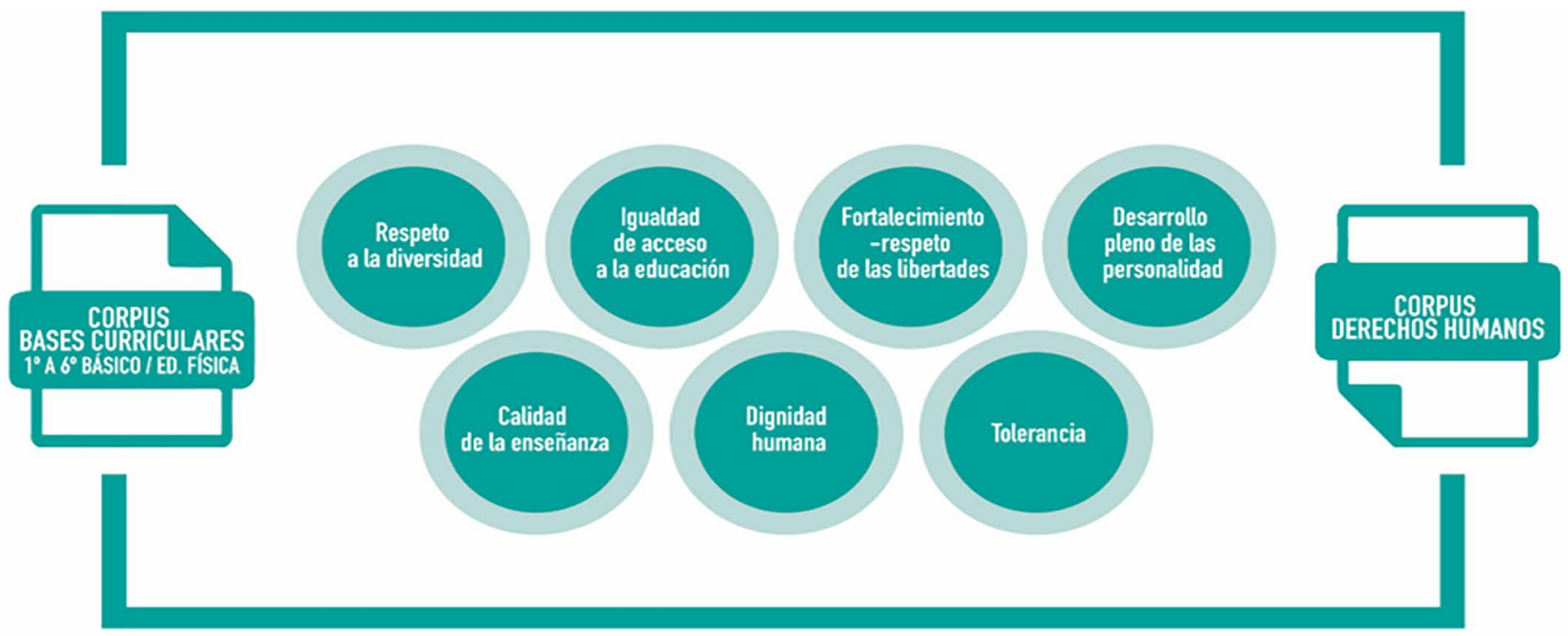

Figure 3. Detalle del diagrama de comparación entre corpus Bases Curriculares y corpus normativo Derechos Humanos Fuente: Producido por los autores

\section{TRIDIMENSIONALIDAD DE DERECHOS HUMANOS}

\section{HECHO HISTÓRICO-CULTURAL}

NORMA

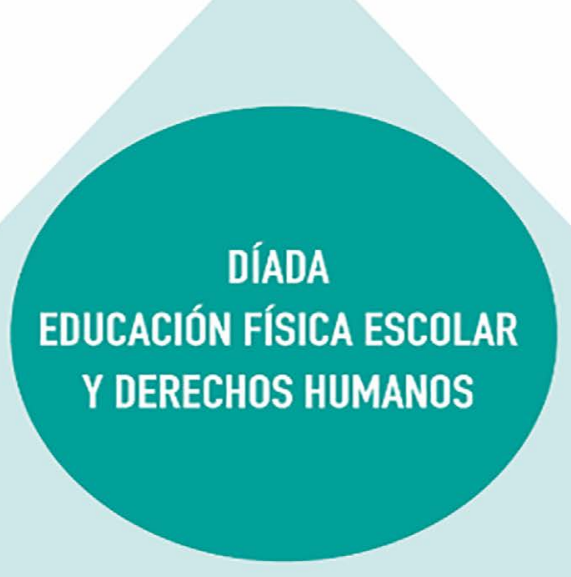

Figure 4. Díada Educación Física Escolar-Derechos Humanos en su matriz. Fuente: Producido por los autores

positivos de la práctica regular de actividad física para la salud; demostrar disposición a mejorar su condición física e interés por practicar actividad física de forma regular; demostrar confianza en sí mismos al practicar actividad física; demostrar disposición a participar de manera activa en la clase; promover la participación equitativa de hombres y mujeres en toda actividad física o deporte; respetar la diversidad física de las personas sin discriminar por características como la altura, peso, color de piel o pelo, etc; demostrar disposición a trabajar en equipo, colaborar con otros y aceptar consejos y críticas; demostrar disposición al esfuerzo personal, superación y perseverancia(MINEDUC, 2018, p. 112). Este conjunto de actitudes se compara con las 7 categorías o nodos principales del diagrama de intersección corpus Derechos Humanos y Bases Curriculares Nacionales en la búsqueda de categorías de Derechos Humanos. Los resultados de esta contrastación permiten concluir que solo 2 actitudes expresan, explícitamente, la promoción de los Derechos Humanos; una, referida a la promoción de la participación y la otra, al respeto de la diversidad. En el trasfondo de las otras 6 actitudes, puede inferirse la presencia de los Derechos Humanos, lo que permite emplazarlas en una dimensión implícita. 


\section{Un hiato en el centro de la díada Educación Física} Escolar-Derechos Humanos. Este hallazgo comunica la distancia, brecha o hiato entre la política curricular declarada en los objetivos de aprendizaje en su eje actitudes, con foco en los Derechos Humanos, entre las Bases Curriculares Nacionales y su concreción en la asignatura de Educación Física y Salud. Las Bases Curriculares Nacionales declaradas se construyen sobre la base de una concepción antropológica y ética emanada desde la Declaración Universal de los Derechos Humanos (MINEDUC, 2018). Al compararlas con el corpus de Derechos Humanos, se observa que su dimensión sociocultural plantea 4 actitudes vinculadas manifiestamente con los Derechos Humanos y su dimensión moral explicita 4 actitudes. En algunos de ellas, los Derechos Humanos están planteados en forma explícita otros, en cambio, pueden ser inferidos desde el plano subyacente. Así, constituye una formulación explícita "Conocer, respetar y defender la igualdad de derechos esenciales de todas las personas, sin distinción de sexo, edad, condición física, etnia, religión o situación económica, y actuar en concordancia del principio ético que reconoce que todos los "seres humanos nacen libres e iguales en dignidad y derechos y, dotados de razón y conciencia, deben comportarse fraternalmente los unos con los otros (Declaración Universal de Derechos Humanos, Artículo 10)" (MINEDUC, 2018, p.30). Ejemplo de una formulación implícita es "Valorar el compromiso en las relaciones entre las personas al acordar contratos: en la amistad, en el amor, en el matrimonio, en el trabajo y al emprender proyectos" (MINEDUC, 2018, p.30).

Al comparar este mismo aspecto, desde lo genérico a lo específico, es decir, desde las Bases Curriculares Nacionales a las Bases Curriculares Nacionales de Educación Física y Salud, es posible reafirmar que, en estas últimas, solo 2 actitudes abordan explícitamente el tema de los Derechos Humanos, vinculados a la diversidad y la participación y en el plano subyacente se descubren las otras 6 actitudes donde puede inferirse alguna presencia de los Derechos Humanos.

Ilustrativo de lo anterior son los documentos de Derechos Humanos estudiados, donde la condena a la discriminación por diversos motivos (étnicos, raciales, religiosos, de género), posee una alta relevancia; es más, si consideramos la categoría aislada de discriminación, esta figura en un 1,78\%, además de expresiones compuestas del tipo discriminación racial, que también tienen una frecuencia de $0,84 \%$. Ello es congruente con la prohibición de la discriminación en el texto de los Derechos Humanos que regula la igualdad de protección ante la ley y prohíbe la discriminación frente al goce de los derechos y libertades (ONU, 1948). Sin embargo, en el corpus de las Bases Curriculares Educación Física y Salud, el discurso sobre la discriminación desconsidera sus aspectos históricos, políticos y socioculturales.

La distancia o hiato entre las intenciones y realizaciones educativas constituye el campo de estudio del currículum (Stenhouse,1991); la díada Derechos Humanos y Educación Física Escolar participa de este hiato. La tridimensionalidad de los Derechos Humanos que enmarca la díada, permite comprender las posibilidades, limitaciones y desafíos de una matriz para la Educación Física Escolar fundada en los Derechos Humanos.

\section{CONCLUSIONES}

El desarrollo del artículo permite arribar a las siguientes conclusiones y proyecciones.

1.El sentido descubierto en la díada entre Educación Física Escolar y Derechos Humanos, es necesario para diseñar, ejecutar y evaluar sus alcances prácticos en el sistema escolar.7 categorías principales representan ese sentido: respeto a la dignidad humana, respeto a la diversidad, igualdad de acceso a la educación, tolerancia, desarrollo pleno de la personalidad, fortalecimiento del respeto de las libertades y calidad de la enseñanza. Este trabajo intenta mostrar la validez de la pregunta por el sentido de la Educación Física Escolar, para diseñar una política curricular para la Educación Física Escolar, máxime en un ámbito como el educativo que refiere a la traducción, reproducción y producción de la cultura en los espacios escolares.

La pregunta por el sentido, es propia de las ciencias del espíritu, unida al intento de comprender la complejidad del fenómeno humano; es posible concluir sobre la validez del diseño cualitativo inscrito en el construccionismo y su metodología hermenéutica /dialéctica, en un artículo, como este, que busca la congruencia entre lo ontológico, epistemológico y metodológico. Precisamente, la ejecución de este diseño permite descubrir, en las categorías de la díada, una dimensión explícita y, otra, implícita, en sintonía con el código curricular visible e invisible u oculto, característico de cualquier institución escolar.

2.La ponderación del sentido de la díada: una pirámide invertida. El estudio del corpus permite concluir que la amplia presencia del corpus de DDHH -estrato superior de la pirámide-se reduce en las Bases Curriculares Nacionales -estrato medio-; y tiene una presencia menor en las Bases Curriculares para Educación Física y Salud -estrato inferior-. Es notable advertir esta disimetría generada en la ejecución del diseño curricular, en un ámbito para el cual se predica la máxima simetría; de las Bases Curriculares Nacionales que evidencian 8 actitudes vinculadas 
explícitamente con Derechos Humanos, en las Bases Curriculares para Educación Física y Salud quedan reducidas a 2 categorías formuladas explícitamente y 6 susceptibles de inferir desde el ámbito subyacente.

\section{Bases Curriculares Nacionales en su circunstancia.} ¿Por qué pierden presencia los Derechos Humanos en la concreción de las Bases Curriculares Nacionales en la asignatura de Educación Física y Salud? El artículo destaca la tensión entre el currículum común y obligatorio y el currículum diferenciado que prescriben las Bases Curriculares Nacionales mediante: tiempo de libre disposición, plan de estudio, y programa de estudio. Esto constituye una fuente que diferencia $y$, a la larga, segrega a establecimientos educacionales, estudiantes y familias. Unido a ello, el artículo enuncia el rol del Estado subsidiario y el modelo de financiamiento que subsidia a la demanda, que genera un sistema educativo que, a contrario sensu del EBDH, se desmarca del derecho a la educación y contribuye a profundizar la desigualdad educativa (Oliva, 2008).

La principal limitación de la investigación es la decisión de trabajar con el currículum prescrito en consonancia con la naturaleza del problema; la política curricular oficial significa y legitima las intenciones de la enseñanza y, a su paso, determina aspectos de financiamiento, la estructura del puesto laboral docente y la política de materiales curriculares, entre otros.

Esta limitación entraña una oportunidad que permite proyectar los alcances de la tesis planteada. El hiato entre intenciones y realizaciones -manifestación de la relación entre teoría y práctica- es un reto para investigar en el currículum en acción, proyectar lo realizado al estudio de las prácticas degestión escolar, de enseñanza-aprendizaje, de materiales curriculares, en fin, de evaluación. Un estudio hipotético-deductivo (cuantitativo) podría cruzar la fuente de datos de la díada con la medición de las actividades desarrolladas para el cumplimiento de los objetivos de aprendizaje de Educación Física y Salud en una pequeña muestra de establecimientos educacionales de distinto nivel de dependencia.

Este es el saber que el presente artículo compromete por la Educación Física Escolar como un Derecho Humano y por el Derecho Humano a una Educación Física.

\section{AYUDA FINANCIERA}

Este trabajo contó con la ayuda financiera de la Agencia Nacional de Investigación y Desarrollo de Chile y el Fondo Nacional de Desarrollo Científico y Tecnológico de Chile [ANID/FONDECYT]. Proyecto de Investigación Fondecyt de Iniciación №11170096 titulado Derecho a la Educación. Enfoque para el Análisis del Discurso de la Calidad de la Educación en la Política Educativa Chilena (1990-2016); los autores agradecen esta ayuda.

\section{CONFLICTOS DE INTERÉS}

Los autores declaran que no hay conflictos de interés.

\section{AGRADECIMIENTOS}

Los autores agradecen a los ayudantes de investigación Andrea Salazar y Gustavo Herrera-Urízar, por su participación en una de las fases de la investigación.

\section{REFERENCIAS BIBLIOGRÁFICAS}

Carta de Toronto para la promoción de la actividad física. [Internet]. 2010. Recuperado a partir de: https://www. fundadeps.org/recursos/documentos/187/Carta-torontoactifisica.pdf

Comité de Derechos Económicos, Sociales y Culturales. [DESC]. Observación General N¹3: El derecho a la educación [Internet]. 1999. Recuperado a partir de http://www.escrnet.org/docs/i/428712.

Consejo de Europa. Carta Europea del Deporte. Hezkuntzako Euskal Erakundea: Instituto Vasco de Educación Física [Internet] 1992. Recuperado a partir de: http://femp.femp. es/files/566-69-archivo/CARTA\%20EUROPEA\%20DEL\%20 DEPORTE.pdf.

Consejo Europeo de Niza. Carta de los Derechos Fundamentales. Niza: Parlamento Europeo [Internet].2000. Recuperado a partir de: http://www.europarl.europa.eu/summits/ nice1_es.htm.

Dilthey, W. Introducción a las ciencias del espíritu. Madrid: Alianza editorial; 2007. $584 \mathrm{p}$.

Fernández Enguita M. La escuela a examen. Madrid: Pirámide; $1997.222 \mathrm{p}$.

Gimeno Sacristán, J. El curriculum: una reflexión sobre la práctica. Madrid: Morata; $1995.423 \mathrm{p}$.

Guba E, Lincoln Y. Competing paradigms in qualitative research. En Denzin N, Lincoln Y, editores. Handbook of qualitative research. California: Thousand Oaks [Internet] 1994. p. 105-117. Recuperado a partir de: https://eclass.uoa.gr/ modules/document/file.php/PPP356/Guba\%20\%26\%20 Lincoln\%201994.pdf.

Habermas J. El concepto de dignidad humana y la utopía realista de los Derechos Humanos. Diánoia [Internet] 2010; 55(64):3-25. Recuperado a partir de: http://www.scielo.org.mx/scielo. php?script=sci_arttext\&pid=S0185-24502010000100001.

Jackson, Ph. W. La vida en las aulas. Madrid: Morata; 1998. 215p.

Lundgren U. Teoría del currículum y la escolarización. Madrid: Morata; 1997. 127 p.

Minsal.Encuesta NacionaldeSalud2016-2017. Primeros resultados. [Internet]. 2017. Recuperado a partir de: http://www.ipsuss. cl/ipsuss/site/artic/20171122/asocfile/20171122142253/ ens_2016_17_primeros_resultados.pdf.

Mineduc. Bases Curriculares Primero a Sexto Básico. [Internet]. 2018. Recuperado a partir de: https://www. curriculumnacional.cl/614/articles-22394_bases.pdf

Mineduc. Ley № 18.962 Ley Orgánica Constitucional de Enseñanza [LOCE] [Internet]. 1990. Recuperada a partir de: https://www.oei.es/historico/quipu/chile/Ley_organica_ ensenanza.pdf.

Mineduc. Ley № 20.370 Ley General de Educación [LGE] [Internet]. 2009. Recuperada a partir de: http://www. movilh.cl/documentos/LGE.pdf. 
Ministerio de Justicia y Derechos Humanos. Subsecretaría de Derechos Humanos. Análisis de aplicación de Enfoque Basado en Derechos Humanos en el Plan Nacional de Derechos Humanos. Santiago: Ministerio de Justicia y Derechos Humanos [Internet] 2018. Recuperado a partir de: http://ddhh.minjusticia.gob.cl/media/2018/08/ANALISIS_ DE_APLICACION_DE_ENFOQUEBASADO_EN_DERECHOS_ HUMANOS_EN_EL_PNDH.pdf .

Moreno A, Campos M, Almonacid A. Las funciones de la educación física escolar: una mirada centrada en la justicia social y la reconstrucción del conocimiento. Estudios Pedagógicos, 2012; 38 (1): 13-26. doi.org/10.4067/S0718-07052012000400002 .

Moreno A, Gamboa R, Poblete C. La educación física en Chile: Análisis crítico de la documentación ministerial. Rev. Bras. Ciênc. Esporte [Internet]. 2014, 36 (2): 411-427. Recuperado a partir de: http://www.scielo.br/pdf/rbce/ v36n2/0101-3289-rbce-36-02-00411.pdf.

Moreno A. La educación física chilena en educación básica: una caracterización crítica. Journal of the Latin American Sociocultural Sudies [Internet]. 2018, 9 (2): 65-78. Recuperado a partir de: http://www.revistas.ufpr.br .

Oficina del Alto Comisionado de las Naciones Unidad para los Derechos Humanos (OACNUDH). Política públicas y presupuestos con perspectivas de Derechos Humanos. Ciudad de México: OACNUDH [Internet]. 2010. Recuperado a partir de: https://www.hchr.org.mx/images/doc_pub/ L280211PPDH.pdf.

Oficina del Alto Comisionado de las Naciones Unidad para los Derechos Humanos (OACNUDH). Preguntas frecuentes sobre el Enfoque de Derechos Humanos en la cooperación para el desarrollo. Nueva York y Ginebra: Naciones Unidas [Internet]. 2006. Recuperado a partir de: https://www. ohchr.org/Documents/Publications/FAQsp.pdf.
Oliva M. Seis epígrafes para la disciplina escolar. Revista Lusófona de Educación, [Internet]. 2019, 43: 11-26. doi: 10.24140/issn.1645-7250.rle43.01.

Oliva M. Arquitectura de la Política Educativa Chilena (19902014): el currículum, lugar de la metáfora. Rev. Bras. Educ., 2017, 22 (69): 405-428. Recuperado a partir de: http:// dx.doi.org/10.1590/s1413-24782017226921.

Oliva M. Política educativa y profundización de la desigualdad en Chile Revista Estudios Pedagógicos, 2008, 34 (2), 207-226.

Organización de las Naciones Unidas [ONU]. Declaración universal de los Derechos Humanos [Internet]. 1948. Recuperada a partir de: http://www.un.org/es/udhrbook/index.shtml.

Organización de las Naciones Unidas para la Educación, la Ciencia y la Cultura. [UNESCOCNA]. Carta Internacional de la Educación Física, la Actividad Física y el Deporte [Internet]. 2015. Recuperado a partir de: file:///C:/Users/ upla/Downloads/Carta-Internacional.pdf.

Reale M. Teoría Tridimensional del Derecho. Madrid: Tecnos; 1997. $176 \mathrm{p}$.

Stenhouse L. Investigación y desarrollo del currículum. Madrid: Morata; 1991.319 p.

Taylor, S.J. y Bogdan, R. Introducción a los métodos cualitativos de investigación. Barcelona: Paidós Ibérica; 1987. 343p.

Tomasevski K. Manual on rights-based education: global human rights requirements made simple. Bangkok: UNESCO [Internet]. 2004. Recuperado a partir de: https://www. right-to-education.org/sites/right-to-education.org/files/ resource-attachments/Manual\%20on\%20Rights-based\%20 Education_Tomasevski_0.pdf.

Varela J. El estatuto del saber pedagógico. En V.V.A.A. Volver a pensar la educación. Madrid: Morata; 1995. p. 61-69.

Valles M. Técnicas cualitativas de investigación social. Madrid: Síntesis; 1999. 432 p. 